\title{
SOME SPECIAL BOUNDARY PROBLEMS IN THE THEORY OF HARMONIC FUNCTIONS.
}

\author{
BY DR. T. H. GRONWALL.
}

(Read before the American Mathematical Society, September 11, 1912.)

1. IN his paper on "Le problème de Dirichlet dans une aire annulaire," Rendiconti del Circolo Matematico di Palermo, volume 33 (1912), pages 134-174, H. Villat has shown that when $u(\rho, \theta)$ is harmonic, uniform, and regular in the circular ring $R>\rho>r$, and subject to the boundary conditions

$$
u(R, \theta)=\Phi(\theta), \quad u(r, \theta)=\Psi(\theta),
$$

$\Phi(\theta)$ and $\Psi(\theta)$ being integrable for $0 \leqq \theta \leqq 2 \pi$ and satisfying the condition

$$
\int_{0}^{2 \pi} \Phi(\theta) d \theta=\int_{0}^{2 \pi} \Psi(\theta) d \theta
$$

necessary for the uniformity of $u(\rho, \theta)$, then this function is given by

$$
\begin{array}{r}
u(\rho, \theta)+i v(\rho, \theta)=\frac{i \omega}{\pi^{2}} \int_{0}^{2 \pi} \Phi(\alpha) \frac{\sigma^{\prime}}{\sigma}\left(\frac{\omega}{i \pi} \log \frac{\rho}{R}+\frac{\omega}{\pi}(\theta-\alpha)\right) d \alpha \\
-\frac{i \omega}{\pi^{2}} \int_{0}^{2 \pi} \Psi(\alpha) \frac{\sigma_{3}{ }^{\prime}}{\sigma_{3}}\left(\frac{\omega}{i \pi} \operatorname{og} \frac{\rho}{R}+\frac{\omega}{\pi}(\theta-\alpha)\right) d \alpha,
\end{array}
$$

where $\sigma$ and $\sigma_{3}$ are the Weierstrass elliptic sigma functions, the periods $\omega$ and $\omega^{\prime}$ satisfying the conditions

$$
\omega \text { and } \frac{\omega^{\prime}}{i} \text { real, } \frac{\omega^{\prime}}{i \omega}=\frac{1}{\pi} \log \frac{R}{r} \text {. }
$$

Villat's proof consists in a somewhat lengthy discussion of the integrals in (3), similar to the ordinary treatment of Poisson's integral.

Fejér* has solved Dirichlet's problem for a circle without the use of Poisson's integral. In the present note, I propose

* L. Fejér, "Untersuchungen über trigonometrische Reihen," Math. Annalen, vol. 58 (1904), pp. 51-69. 
to extend Fejér's process to obtain Villat's results (and the corresponding ones in three-dimensional space) in a considerably shorter way than by the method referred to.

2. We first notice that, $\Phi(\theta)$ and $\Psi(\theta)$ being integrable, their Fourier coefficients

$$
\begin{aligned}
& a_{n}=\frac{1}{\pi} \int_{0}^{2 \pi} \Phi(\alpha) \cos n \alpha d \alpha, \quad b_{n}=\frac{1}{\pi} \int_{0}^{2 \pi} \Phi(\alpha) \sin n \alpha d \alpha, \\
& a_{n}{ }^{\prime}=\frac{1}{\pi} \int_{0}^{\pi} \Psi(\alpha) \cos n \alpha d \alpha, \quad b_{n}{ }^{\prime}=\frac{1}{\pi} \int_{0}^{\pi^{*}} \Psi(\alpha) \sin n \alpha d \alpha
\end{aligned}
$$

exist, which fact we denote by

$$
\begin{aligned}
& \Phi(\theta) \sim \frac{a_{0}}{2}+\sum_{n=1}^{\infty}\left(a_{n} \cos n \theta+b_{n} \sin n \theta\right), \\
& \Psi(\theta) \sim \frac{a_{0}{ }^{\prime}}{2}+\sum_{n=1}^{\infty}\left(a_{n}{ }^{\prime} \cos n \theta+b_{n}{ }^{\prime} \sin n \theta\right),
\end{aligned}
$$

the equivalence sign $\sim$ implying no statement whatever in regard to the convergence of the right-hand members. We now assume a development

$$
\begin{aligned}
u(\rho, \theta)=\frac{A_{0}}{2}+\sum_{n=1}^{\infty}\left(A_{n}\right. & \left.\cos n \theta+B_{n} \sin n \theta\right) \rho^{n} \\
& +\sum_{n=1}^{\infty}\left(C_{n} \cos n \theta+D_{n} \sin n \theta\right) \rho^{-n}
\end{aligned}
$$

make $\rho=R$ and $\rho=r$, and identify the resulting Fourier series, term by term, with the Fourier series (5) for $\Phi(\theta)$ and $\Psi(\theta)$ respectively, which gives the equations

$$
\begin{aligned}
A_{0}=a_{0}=a_{0}{ }^{\prime}, & \\
A_{n} R^{n}+C_{n} R^{-n}=a_{n}, & B_{n} R^{n}+D_{n} R^{-n}=b_{n}, \\
A_{n} r^{n}+C_{n} r^{-n}=a_{n}{ }^{\prime}, & B_{n} r^{n}+D_{n} r^{-n}=b_{n}{ }^{\prime} \\
& \quad(n=1,2,3, \cdots) .
\end{aligned}
$$

The two equations for $A_{0}$ are consistent by (2), and from the others we obtain, making $r=q R$, so that $q<1$,

$$
\begin{array}{ll}
A_{n}=\frac{R^{-n}}{q^{-n}-q^{n}}\left(a_{n} q^{-n}-a_{n}{ }^{\prime}\right), & B_{n}=\frac{R^{-n}}{q^{-n}-q^{n}}\left(b_{n} q^{-n}-b_{n}{ }^{\prime}\right), \\
C_{n}=\frac{R^{n}}{q^{-n}-q^{n}}\left(a_{n}{ }^{\prime}-a_{n} q^{n}\right), & D_{n}=\frac{R^{n}}{q^{-n}-q^{n}}\left(b_{n}{ }^{\prime}-b_{n} q^{n}\right) .
\end{array}
$$


To investigate the convergence of (6), we note that, when $n$ is large enough to make $q^{2 n}<\frac{1}{2}$,

$$
\begin{aligned}
& \left|A_{n}\right|=R^{-n}\left|\frac{a_{n}-a_{n}{ }^{\prime} q^{n}}{1-q^{2 n}}\right|<2 R^{-n}\left(\left|a_{n}\right|+\left|a_{n}{ }^{\prime}\right|\right), \\
& \left|B_{n}\right|=R^{-n}\left|\frac{b_{n}-b_{n}{ }^{\prime} q^{n}}{1-q^{2 n}}\right|<2 R^{-n}\left(\left|b_{n}\right|+\left|b_{n}{ }^{\prime}\right|\right), \\
& \left|C_{n}\right|=R^{n} q^{n}\left|\frac{a_{n}{ }^{\prime}-a_{n} q^{n}}{1-q^{2 n}}\right|<2 r^{n}\left(\left|a_{n}\right|+\left|a_{n}{ }^{\prime}\right|\right), \\
& \left|D_{n}\right|=R^{n} q^{n}\left|\frac{b_{n}{ }^{\prime}-b_{n} q^{n}}{1-q^{2 n}}\right|<2 r^{n}\left(\left|b_{n}\right|+\left|b_{n}{ }^{\prime}\right|\right) .
\end{aligned}
$$

Now, $\Phi(\theta)$ and $\Psi(\theta)$ being integrable, $\frac{1}{n} a_{n}, \frac{1}{n} b_{n}, \frac{1}{n} a_{n}{ }^{\prime}, \frac{1}{n} b_{n}{ }^{\prime}$ are bounded for all values of $n, *$ and therefore it follows from (9) that both series in (6), together with their partial derivatives term by term to any order, are uniformly convergent, the first for $\rho \leqq R-\epsilon, 0 \leqq \theta \leqq 2 \pi$, and the second for $\rho \geqq r+\epsilon, 0 \leqq \bar{\theta} \leqq 2 \pi$, where $\epsilon$ is positive and as small as we like.

Hence the expression (6) is harmonic, uniform, and regular for $R>\rho>r$. To show that the boundary conditions (1) are satisfied, we shall use the following lemma: $\dagger$ Let

$$
F(x, \theta)=\sum_{n=0}^{\infty} u_{n}(\theta) x^{n},
$$

and suppose that the (eventually divergent) series

$$
\sum_{n=0}^{\infty} u_{n}(\theta)
$$

is summable by Cesàro's mean values of the first order, with the sum $s(\theta)$ (that is, the limit

$$
\lim _{n=\infty} \frac{1}{n+1} \sum_{\mu=0}^{n} \sum_{\nu=0}^{\mu} u_{\nu}(\theta)=s(\theta)
$$

\footnotetext{
* Fejér, l. c.
}

$\dagger$ Fejér, 1. c., $\S 2$, specializing his general theorem by making $t=\log (1 / x)$, $\varphi(t)=e^{-t}$. Compare also G. H. Hardy, "Some theorems concerning infinite series," Math. Annalen, vol. 64 (1907), pp. 77-94. 
does exist); then (10) is convergent for $0 \leqq x<1$ and

$$
\lim _{h=+0} F(1-h, \theta)=s(\theta),
$$

and on any range for $\theta$ where (11) holds uniformly the same is true of (12).

Now the Fourier series (5) for $\Phi(\theta)$ is uniformly summable of the first order with the sum $\Phi(\theta)$ on any range where $\Phi(\theta)$ is continuous.* Furthermore, the series

$$
\Phi_{1}(\theta)=\sum_{n=1}^{\infty}\left(C_{n} \cos n \theta+D_{n} \sin n \theta\right) R^{-n},
$$

being uniformly convergent, is obviously uniformly summable of the first order with the sum $\Phi_{1}(\theta)$; hence, by (7), the series

$$
\frac{A_{0}}{2}+\sum_{n=1}^{\infty}\left(A_{n} \cos n \theta+B_{n} \sin n \theta\right) R^{n}
$$

is uniformly summable of the first order with the sum $\Phi(\theta)$ $-\Phi_{1}(\theta)$. Making $x=\rho / R$ and applying the lemma, it is seen that

(13) $\lim _{h=+0} u(R(1-h), \theta)=\Phi(\theta)-\Phi_{1}(\theta)+\Phi_{1}(\theta)=\Phi(\theta)$

uniformly on any range where $\Phi(\theta)$ is continuous. Making $x=r / \rho$ and reasoning in the same way on $\Psi(\theta)$, we obtain

$$
\lim _{h=+0} u\left(\frac{r}{1-h}, \theta\right)=\Psi(\theta)
$$

uniformly on any range where $\Psi(\theta)$ is continuous, so that our expression (6) satisfies all the conditions of the problem.

Introducing the values (4) into (8) and using well known formulæ in elliptic functions, we may easily reduce (6) to the form (3).

3. Now suppose that $\Phi(\theta)$ is continuous for $\theta=\theta_{0}$, and let the point $\rho, \theta$ approach $R, \theta_{0}$ along any continuous path $\rho=\rho(\theta)$ instead of the radius. For any positive $\epsilon$ we may find a $\delta$ such that, $\Phi(\theta)$ being continuous for $\theta=\theta_{0}$,

$$
\left|\Phi(\theta)-\Phi\left(\theta_{0}\right)\right|<\frac{1}{2} \epsilon \text { for } \theta_{0}-\delta<\theta<\theta_{0}+\delta .
$$

\footnotetext{
* Fejér, l. c., § 1 .
} 
By (13), there exists an $\eta$ independent of $\theta$ such that

$$
\begin{aligned}
& |u(R(1-h), \theta)-\Phi(\theta)|<\frac{1}{2} \epsilon \\
& \quad \text { for } 0<h \leqq \eta \text { and } \theta_{0}-\delta<\theta<\theta_{0}+\delta,
\end{aligned}
$$

and according to the definition of $\rho(\theta)$ there exists a $\delta^{\prime}<\delta$ such that

$$
0<R-\rho(\theta)<R \eta \text { for } \theta_{0}-\delta^{\prime}<\theta<\theta_{0}+\delta^{\prime} .
$$

The combination of these inequalities gives

$$
\left|u(\rho(\theta), \theta)-\Phi\left(\theta_{0}\right)\right|<\epsilon \text { for } \theta_{0}-\delta^{\prime}<\theta<\theta_{0}+\delta^{\prime},
$$

so that, for any $\theta_{0}$ where $\Phi(\theta)$ is continuous, $u(\rho, \theta)$ tends towards $\Phi\left(\theta_{0}\right)$ when $\rho, \theta$ approaches $R, \theta_{0}$ by any continuous path entirely inside the circle of radius $R$.

The case of a point of discontinuity $\theta_{0}$, such that $\Phi\left(\theta_{0}+0\right)$ and $\Phi\left(\theta_{0}-0\right)$ both exist, may be reduced to the continuity case, according to H. A. Schwarz, by replacing $u(\rho, \theta)$ by

$$
u(\rho, \theta)-\frac{\Phi\left(\theta_{0}+0\right)-\Phi\left(\theta_{0}-0\right)}{\pi} \operatorname{arctg} \frac{\rho \sin \theta-R \sin \theta_{0}}{\rho \cos \theta-R \cos \theta_{0}},
$$

and from the preceding results, all of Villat's theorems are easily deduced.

4. Passing to three-dimensional space referred to polar coordinates, consider the harmonic function $u(p, \theta, \varphi)$, uniform and regular for $r<\rho<R$, and with the boundary conditions

$$
u(R, \theta, \varphi)=\Phi(\theta, \varphi), \quad u(r, \theta, \varphi)=\Psi(\theta, \varphi),
$$

where $\Phi$ and $\Psi$ are integrable on the unit sphere.

We then have the formal developments in spherical harmonics

$$
\Phi(\theta, \varphi) \sim \sum_{n=0}^{\infty} Y_{n}^{\prime}(\theta, \varphi), \quad \Psi(\theta, \varphi) \sim \sum_{n=0}^{\infty} Y_{n}^{\prime \prime}(\theta, \varphi),
$$

and assuming a development

$$
u(\rho, \theta, \varphi)=Y_{0}+\sum_{n=1}^{\infty} Y_{n}(\theta, \varphi) \rho^{n}+\sum_{n=1}^{\infty} \bar{Y}(\theta, \varphi) \rho^{-n},
$$

we make $\rho=R$ and $\rho=r$ and identify (16) term by term 
with the developments of $\Phi$ and $\Psi$ respectively, obtaining

whence

$$
\begin{aligned}
Y_{0}=Y_{0}^{\prime} & =Y_{0}^{\prime} \quad \quad \text { (condition of uniformity), } \\
R^{n} Y_{n}+R^{-n} \bar{Y}_{n} & =Y_{n}^{\prime}, \\
r^{n} Y_{n}+r^{-n} \bar{Y}_{n} & =Y_{n}^{\prime \prime} \quad(n=1,2,3, \cdots),
\end{aligned}
$$

$$
\begin{aligned}
& Y_{n}=\frac{R^{-n}}{q^{-n}-q^{n}}\left(q^{-n} Y_{n}^{\prime}-Y_{n}^{\prime \prime}\right), \\
& \bar{Y}_{n}=\frac{q^{n} R^{n}}{q^{-n}-q^{n}}\left(q^{-n} Y_{n}^{\prime \prime}-Y_{n}^{\prime}\right),
\end{aligned}
$$

By expressing $Y_{n}^{\prime}$ and $Y_{n}^{\prime \prime}$ by finite trigonometric sums in the usual way and slightly modifying Fejér's argument for showing the boundedness of $a_{n} / n, \cdots, b_{n}{ }^{\prime} / n$ in (8), it is readily seen that $Y_{n}^{\prime} / n^{4}$ and $Y_{n}^{\prime \prime} / n^{4}$ are bounded, and the convergence of (16) and its partial derivatives term by term is then established exactly as that of (6). The expression (16) is therefore harmonic, uniform, and regular for $r<\rho<R$; to prove that the boundary conditions are satisfied, we may proceed in the same way as before, only substituting Cesàro's means of the second order for those of the first order previously used.*

Introducing the well-known integral expressions for $Y_{n}{ }^{\prime}$ and $Y_{n}^{\prime \prime}$, we may write (16) in the form

$$
\begin{aligned}
u(\rho, \theta, \varphi) & =\frac{1}{4 \pi} \int_{0}^{\pi} \int_{0}^{2 \pi} \Phi(\alpha, \beta)\left\{1+\sum_{n=1}^{\infty} \frac{2 n+1}{q^{-n}-q^{n}}\left[\left(\frac{\rho}{q R}\right)^{n}\right.\right. \\
& \left.\left.-\left(\frac{\rho}{q R}\right)^{-n}\right] P_{n}(\cos \gamma)\right\} \sin \alpha d \alpha d \beta \\
& -\frac{1}{4 \pi} \int_{0}^{\pi} \int_{0}^{2 \pi} \Psi(\alpha, \beta) \sum_{n=1}^{\infty} \frac{2 n+1}{q^{-n}-q^{n}}\left[\left(\frac{\rho}{R}\right)^{n}\right. \\
& \left.-\left(\frac{\rho}{R}\right)^{-n}\right] P_{n}(\cos \gamma) \sin \alpha d \alpha d \beta,
\end{aligned}
$$

* In the general case of summability of $\sum_{n=0}^{\infty} u_{n}(\theta)$ by Cesàro's means of the $r$ th order, the lemma was proved by $\stackrel{n=0}{H}$ ardy, 1. c. The summability of the second order of the development of a function $f(\theta, \varphi)$ in spherical harmonics was proved by Fejér, "Ueber die Laplacesche Reihe," Math. Annalen, vol. 67 (1909), pp. 76-109, when $f(\theta, \varphi)$ is absolutely integrable, and by the present writer (in a paper with the same title which will appear presently in the Math. Annalen), when $(f(\theta, \varphi)$ is any integrable fnnction. In the same paper, it is shown that for an absolutely integrable function, already the means of the first order are summable. 
$\gamma$ being the angle between the directions $\theta, \varphi$ and $\alpha, \beta$. By using the Mehler formulæ for Legendre's polynomials $P_{n}$, (18) may be transformed so as to contain elliptic sigma functions under a triple integral sign, giving a formula somewhat similar to (3).

Chicago, Ill.,

November $9,1912$.

\section{NOTE ON FERMAT'S LAST THEOREM.}

By PROFESSOR R. D. CARMiChaEL.

(Read before the American Mathematical Society, December 31, 1912.)

THE object of this note is to prove the following

Theorem. If $p$ is an odd prime and the equation

$$
x^{p}+y^{p}+z^{p}=0
$$

has a solution in integers $x, y, z$ each of which is prime to $p$, then there exists a positive integer $s$, less than $\frac{1}{2}(p-1)$, such that

$$
(s+1)^{p^{2}} \equiv s^{p^{2}}+1 \bmod p^{3} .
$$

The proof is elementary. If there exists a set of integers $x, y, z$ satisfying (1), there exists such a set having the further property that they are prime each to each. Consequently, for the purpose of argument we may assume that $x, y, z$ have this property.

Then from elementary considerations it is known* that integers $\alpha, \beta, \gamma$ exist such that

$$
x+y=\gamma^{p}, \quad y+z=\alpha^{p}, \quad z+x=\beta^{p} .
$$

Therefore

(2) $(x+y)^{p-1} \equiv 1,(y+z)^{p-1} \equiv 1,(z+x)^{p-1} \equiv 1 \bmod p^{2}$, since $a^{p(p-1)} \equiv 1 \bmod p^{2}$ when $a$ is prime to $p$.

From (1) it follows that

$$
x+y+z \equiv 0 \bmod p,
$$

* See, for instance, Bachmann's Niedere Zahlentheorie, Zweiter Teil, p. 467 . 